\title{
Modelling of layer-by-layer metal removal in the digital twin of the round grinding process performed on CNC machines
}

\author{
Aleksandra Akintseva*, Pavel Pereverzev, Alexander Prokhorov, and Sergey Kononov \\ South Ural State University, Department of Engineering and Technology, 454080 Chelyabinsk, Lenin \\ Avenue 76, Russia
}

\begin{abstract}
Parts' processing on circular grinding machines is carried out according to the specified stepwise control cycles of radial and axial feeds, which are laid down in the control program $(\mathrm{CP})$. Despite the precise positioning systems, modern $\mathrm{CNC}$ circular grinding machines have a variable compliance of the technological system along the processing length. It causes different cutting depth and occurrence of various errors in dimensions of the diametric surface. Also, the dimensions error increases due to the fact that the processing of parts batch is carried out in unstable conditions and under the influence of different variable factors, which can include allowance fluctuation, blunting of the wheel grains, changes of the wheel diameter and the contact area of the wheel with the workpiece, etc. However, there are still no means of control developed CP, which include cycles of cutting modes, to ensure the accuracy of parts batch processing. This article describes the digital twin (DT) of the circular plunge grinding with $\mathrm{CNC}$, which allows simulating layer-by-layer metal removal during the whole grinding cycle by calculating the cutting depth on each workpiece revolution under various combinations of unstable processing conditions and variable compliance of TS. Modeling of layer-by-layer allowance removal is performed in several sections of the treated surface; these sections have different compliance. At the end of the grinding cycle in the DT the processing accuracy is estimated on the basis of the obtained values of radii dimensions in all sections of the processed surface. The introduction of DT allows speeding up the CP development, reduce the time of launching $\mathrm{CP}$ in production, and increase the productivity of circular grinding operations with $\mathrm{CNC}$ by optimizing the cycles of the cutting modes while ensuring the specified processing accuracy.
\end{abstract}

\section{Introduction}

$\mathrm{CNC}$ circular grinding operations are the main operations of parts finish processing in automated mechanical engineering. Modern grinding machines have technical capabilities that allow processing various external and internal surfaces of the part in one installation of the workpiece [1]. At the same time, there is a need for monthly development the plenty of

\footnotetext{
* Corresponding author: akintsevaav@susu.ru
} 
control programs for circular grinding operations with CNC. However CAD/CAM-systems have no package for calculating optimal cutting modes in conditions of real automated production and for this reason cutting modes for all types of circular grinding operations with $\mathrm{CNC}$ are assigned manually by the technologist. At that the technologist relies on personal experience or on the enterprise`s statistical data on cutting modes in similar operations.

The assignment of cutting modes for circular grinding operations is a difficult task because of the need to develop stepwise cycles of program feeds which are used for the surface treatment. For each program feed cycle it is necessary to assign the number of cycle stages, distribute the removed allowance on the cycle stages and assign the program feed and other cutting modes to each stage. At the same time the technologist must ensure the maximum productivity (or minimum cost price) of the operation with simultaneous ensuring the specified processing accuracy and other parameters of treated surface quality under the influence of different variable technological factors that occur in the processing the batch of parts. These factors include different compliance of the technological system (TS) along the processing length, fluctuation of allowance and radial runout of the workpiece in the parts batch, blunting of the grinding wheel grains, diametrical wear of the wheel and change of the contact area of the wheel with the workpiece during the grinding process, etc. In addition, the same control program (CP) sets the grinding cycles of various external and internal surfaces (cylinder, end, cone) and synchronizes the cycles of various feeds (for example, radial and axial feed cycles). It should be noted that the control of stepwise grinding cycle is carried out by an active control device (ACD) which continuously measures the diameter of the part during grinding and switches program feeds in dependence on the remaining allowance.

Thus, in modern automated production, considering the absence of an optimal cycles designing tool in existing CAD/CAM-systems, thousands CPs are developed monthly for circular grinding operations with $\mathrm{CNC}$. When developing these CPs, the technologist manually assigns control cycles for mode parameters for circular grinding operations with various initial data (parameters of the treated surface, machine parameters, parameters of the grinding wheels characteristic, parameters of the parts batch). It should be noted that the technologist does not have the tools to check if developed CPs ensure the specified accuracy and other parameters of the treated surface quality. Therefore, the current task is to develop a tool for CAD/CAM-systems (software package). The main task of this tool will be control and optimization of $\mathrm{CP}$ for all types of circular grinding operations with $\mathrm{CNC}$ in the field of assigning cutting modes and stepwise cycles of program feeds that ensure the specified accuracy, roughness and hardness of the treated surface in unstable conditions of processing the batch of parts.

Despite the fact that $\mathrm{CNC}$ circular grinding machines have been produced for several decades, it is still a difficult and unsolved scientific and technical task to predict the processing accuracy in CNC circular grinding operations in which program feeds are changed in an automatic stepwise cycle. The reason is in fact that the prediction of processing accuracy in an automatic grinding cycle with switching feeds' program speeds cannot be performed using a one formula because the treated surface is repeatedly formed with forces and cutting depths changing at each revolution along the entire length of the treated surface under the influence of unstable technological factors. Many scientists have worked on above problem; there are some works which can be distinguished [2-10].

The solution of the above problem is possible only with the help of the digital twin (DT) of the circular grinding operation, in which the layer-by-layer allowance removal is modeled by calculating the cutting depth in different sections of the treated surface on each revolution of the workpiece with consideration the influence of variable factors. In the DT the control of $\mathrm{CP}$ is performed to ensure the processing accuracy as follows: at the end of 
the grinding cycle, when the allowance removal is completed, the dimensions of the treated surface in all sections are determined. Design sizes of the treated surface are compared with permissible sizes values on the part drawing and then the dimensional model of the treated surface is constructed and used for calculating the predicted values of errors of the diametric size in different sections and the predicted values of the form deviation and surfaces location.

The creation of the DT became possible as a result of the implementation of a physical and mathematical model of the allowance removal by system modeling of a plenty of interrelated wide-range analytical models of the circular grinding processes (model of elastic deformations of the technological system (TS), cutting force model [11], model of the interrelation between program and actual feeds [12], model of the processing error formation [13], model of the treated surface' dimensions formation [14], etc.). These models are combined into a unified analytical model for calculating the values of actual feeds in several sections of the treated surface on each revolution of the workpiece during the entire grinding cycle for specified grinding conditions under various combinations of unstable factors values when processing the parts batch [15].

Let us consider the concept of the easiest way to use the DT to control the grinding cycle on the operation of the circular external plunge grinding with CNC.

When monitoring the grinding cycle for the accuracy, the use of the DT is carried out in the following stages:

1) input of initial data on the treated part, machine parameters and wheel characteristic, grinding cycle parameters (number of steps of the program radial feeds and their values for each stage, distribution of the removed allowance for the stages), etc.;

2) calculation of the TS compliance values in different cross-sections of the workpiece evenly distributed over the treated surface;

3) selection of the workpiece section, controlled by the ACD, which is essentially used to control the cycle;

4) determination of the number of scenarios for the action of variable technological factors and their numerical values, at which the grinding cycle will be tested in the DT on ensuring the processing accuracy;

5) modeling of layer-by-layer metal removal in a grinding cycle for the specified scenario;

6) determination of the diametric sizes of the workpiece in different sections along the entire length at the end of the grinding cycle;

7) calculation the processing error;

8) checking of the designed cycle for all specified scenarios of action of variable technological factors (repeat points 5-7);

9) determination of the error dispersion when processing the parts batch;

10) generation a conclusion about the grinding cycle fitness and preparation predictive values of the processing accuracy in the form of a document «Certificate of the grinding cycle processing accuracy».

Due to the limited scope of the article, only a part of the main stages will be considered in more detail on the example of circular plunge external grinding.

\section{Modelling of layer-by-layer metal removal in the digital twin of the round grinding process performed on CNC machines}

Variable compliance of the TS along the length of the treated surface is the most important factor that directly affects the processing accuracy. Due to this factor there is an uneven deflection of the workpiece segments about the grinding wheel under the action of the 
cutting force, what determines different cutting depth, and therefore different radii values and errors in dimensions, shape and location all over the treated surface.

There is a method of the TS compliance calculation used in the DT which allows to calculate elastic movement in the machine' TS and assess their influence on the dimensions of the treated surface and processing accuracy. Figure 1, a shows the calculated scheme of the force loading of the shaft for the circular plunge grinding with overall dimensions. The shaft is fixed in non-rigid headstock and tailstock centers with different compliance. Therefore, the shaft supports, which are the centers of the machine, are shown in the scheme as springs, which mean that the supports are non-rigid and can make elastic movements under the influence of a force load.

Radial cutting force $P_{y}$, which occur during the circular plunge grinding, is shown in fig. 1 as a distributed load $q$, acting on the shaft along the length of the treated surface $\mathrm{B}$, from which reactions of the supports $R_{A}$ and $R_{B}$ occur. The force load on the shaft and reactions of the supports cause elastic deformations of the shaft itself, as well as elastic deformations of the headstock and tailstock centers of the machine. TS compliance diagram $\gamma_{c}$ shown in fig. $1, \mathrm{~b}$, is calculated by using standard methods taken from the strength of materials course. As it can be seen from fig. 1, b, TS compliance $\gamma_{c}$ for this calculated scheme varies from the minimum to the maximum value on the length of the treated surface. Therefore, it can be assumed that we get a conical surface after processing.

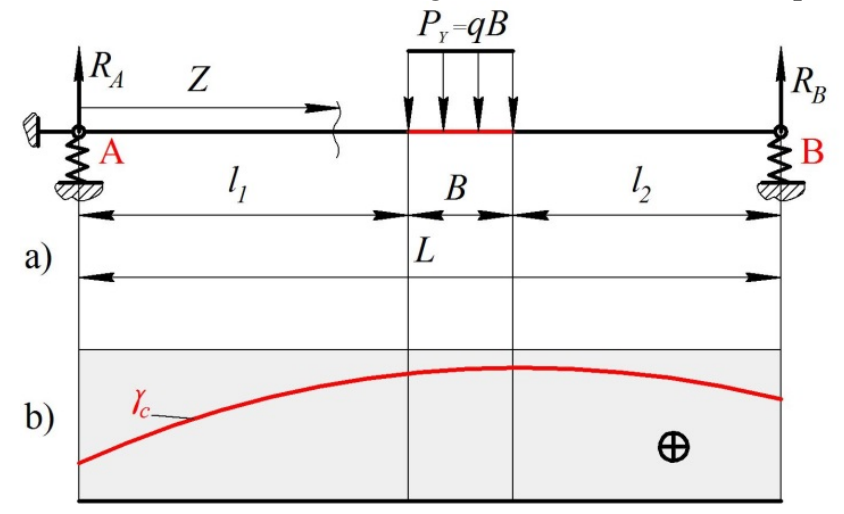

Fig. 1. Scheme of force loading and TS compliance diagram for the circular plunge grinding: (a) scheme of force loading of the shaft; (b) - TS compliance diagram

On modern $\mathrm{CNC}$ machines processing is carried out using the $\mathrm{ACD}$, which during the entire grinding cycle measures the workpiece treated diameter in one section and issues commands to stepwise change of the program feed in dependence on the remained allowance part.

Processing error depends on the choice of the section in which the ACD is installed. If the ACD is installed in the section with the minimum compliance, then in all sections along the processing length the diametric dimensions will be larger than in the section where the ACD is installed. Therefore, there may be an error in the diametric size of the upper limit drawing size. And conversely, if the ACD is installed in the section with the maximum compliance, the diameter size can go beyond the lower permissible limit in the section with minimum compliance. If the ACD is installed in the section with average compliance, the diameters will fluctuate upward and downward. It should be noted that the choice of the ACD section also depends on the presence of discontinuous surface, which include splined surfaces, key slots, etc.

Figure 2 shows the distribution scheme of the four shaft sections along the processing length. The sections are distributed evenly along the length of the treated surface. Section 1 
is located on the left part of the distributed load, in which the compliance $\gamma_{1}$ has the minimum value, i.e. $\gamma_{1}=\gamma_{\min }$ in accordance with the TS compliance diagram in fig. 1 . Section 3 is located on the right part of the distributed load, in which the compliance $\gamma_{3}$ has the maximum value, i.e. $\gamma_{3}=\gamma_{\max }$. Section 2 is located in the middle of the disturbed load and has the average compliance $\gamma_{2}$. Between sections 2 and 3 there is a section with the ACD and the compliance in this section is equal to $\gamma_{\Pi A K}$, that is $\gamma_{2}<\gamma_{A C D}<\gamma_{3}$. Since compliances in sections 1,2 and 3 are not equal to the compliance in the section with the ACD, the diameters in all four sections will be different. Therefore, in sections 1 and 2 the shaft diameters will be less than the diameter in the section with the ACD, and the shaft diameter in the section 3 will be greater. Therefore the diametric error in different sections will be also different. Let us prove these statements on the allowance removal model.

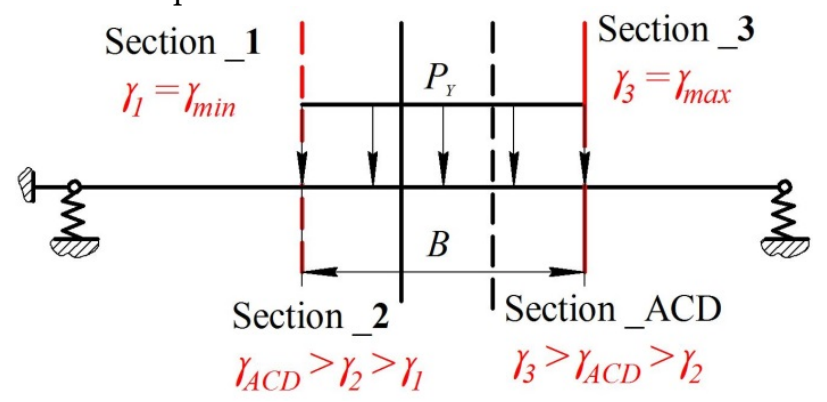

Fig. 2. Calculated scheme of the shaft loading and distribution of the technological system compliance by the section of the treated surface on the site B

The calculation of layer-by-layer metal removal in the circular plunge grinding cycle is resolved to the calculation of the cutting depth per one workpiece revolution, which further will be called the speed of actual feed $\Delta t_{f}$ (we consider it on the example of the circular plunge external grinding). Due to the fact that the TS compliance in different sections of the treated surface is unequal, the speed of actual feed will be also different. It should be noted that the circular grinding operation with $\mathrm{CNC}$ is controlled by a stepwise change of the speed of program feed $\Delta t_{p}$ in the grinding cycle of a single part. The value $\Delta t_{p}$ is equal for all sections of the treated surface, but the values $\Delta t_{f}$ will be different due to the uneven TS compliance along the processing length.

Figure 3, a shows a graph of a two-stage grinding cycle with the program speeds of the feeds $\Delta t_{p_{-} l}$ and $\Delta t_{p_{-} 2}$ at the first and second stage accordingly. Graphs of the speeds of actual feeds $\Delta t_{f_{-} l}, \Delta t_{f_{-} 2}, \Delta t_{f_{-} 3}$ are shown for sections 1,2 and 3 ; the graph $\Delta t_{f_{-} A C D}$ is shown for the section with the ACD. The calculation of the speeds of actual feeds is performed according to the method [14], in which the interconnection of program and actual speeds of the feeds with the TS compliance, cutting force and technological conditions of grinding.

On the basis of the graph of program and actual feeds speeds (fig. 3, a), the graph of the accumulated program feed $t_{p}$ (fig. $3, \mathrm{~b}$ ) and graphs of the actual feeds $t_{f}$ in each shaft section of the treated surface are constructed. Graphs of the actual feeds speeds $t_{f_{-} 1}, t_{f_{-} 2}, t_{f_{-} 3}$ are shown for the sections 1,2 and 3, and the graph $t_{f_{-} A C D}$ is shown for the section with the ACD (fig. 3, b). Graphs of the accumulated program feed $\Delta t_{p_{-} l}$ and $\Delta t_{p_{-} 2}$ are shown for stages 1 and 2 accordingly. Thus, the graphs of the accumulated program and actual feeds in each section of the treated surface are obtained (fig. 3, b). The difference between accumulated actual feeds on the current and previous workpiece revolutions is equal to the cutting depth or actual speed of the feed per a workpiece revolution. Therefore if we construct the graphs of the accumulated actual feeds in each section along the processing length (fig. 4 and 5) by superimposing the graphs on each other with overlap, we'll get the graphs of allowance layers that are removed during the grinding cycle. 
Figure 4 shows a three-dimensional graph of the accumulated actual feed constructed on each workpiece revolution in four shaft sections. The axes of this graph are the accumulated actual feeds $t_{f_{-} l}, t_{f_{-} 2}, t_{f_{-} 3}, t_{f_{-} A C D}$ in four sections, length of the treated surface B and number of the workpiece revolution $N$. The graphs of the accumulated actual feeds meet the graphs of the same name feeds in fig. 3, b. The height differences of the graphs meet the cutting depth along the length of the treated surface.

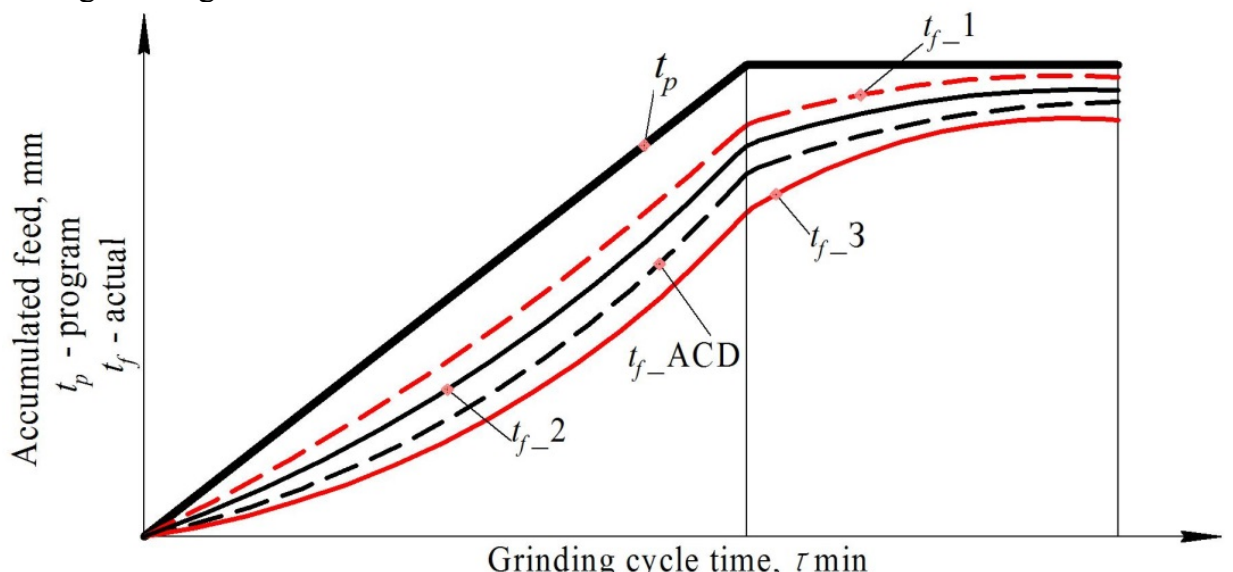

Fig. 3. Graphs of accumulated program and actual feeds for the two-stage grinding cycle

Considering the fact that the graphs of accumulated actual feeds are additive and do not decrease over time, when they are projected on each other we get the graphs of metal layers (fig. 5), removed along the length of the treated surface on each revolution of the workpiece. Each layer, shown in fig. 5, meets the cutting depth per the workpiece revolution. The first bottom layer is removed on the first workpiece revolution. The last top layer is removed on the last workpiece revolution. The summary height of the layer characterizes the value of the removed allowance over the time of grinding in different sections of the shaft. To connect the removed allowance and the shaft dimensions, the upper part of the graph shows the part axis and the radii at the beginning and ending of the grinding cycle.

To calculate the processing error, it is necessary to compare the dimensions of the part after processing with the permissible values by the drawing. Figure 5 shows the part radius' tolerance zone by the drawing. The radii of the finished surface, shown in fig. 5, change within the last removed layer of metal, because during the circular plunge grinding the removal of the metal layer is carried out in a spiral.

The diameter of the finished part in the ACD section is set to the coordinate of the middle of the part tolerance zone. As it can be seen from the example in fig. 5 only the radii of section 2 and $A C D$ section are in the tolerance. The radii in section 1 exceeded the upper permissible limit, and the radii in section 3 exceeded the lower permissible limit. The longitudinal error is a taper that can be easily estimated from the calculated graph.

The graphs of the layer-by-layer metal removal, shown in fig. 5, meet the one scenario of grinding conditions. In the DT the graphs of layer-by-layer metal removal for a plenty of different processing scenarios for the parts batch are calculating. Therefore the scatter of dimensions and errors of the finished surfaces will be much greater. After calculating in the DT the processing error for the entire set of grinding scenarios, a prediction for the processing accuracy for the tested grinding cycle is given. 


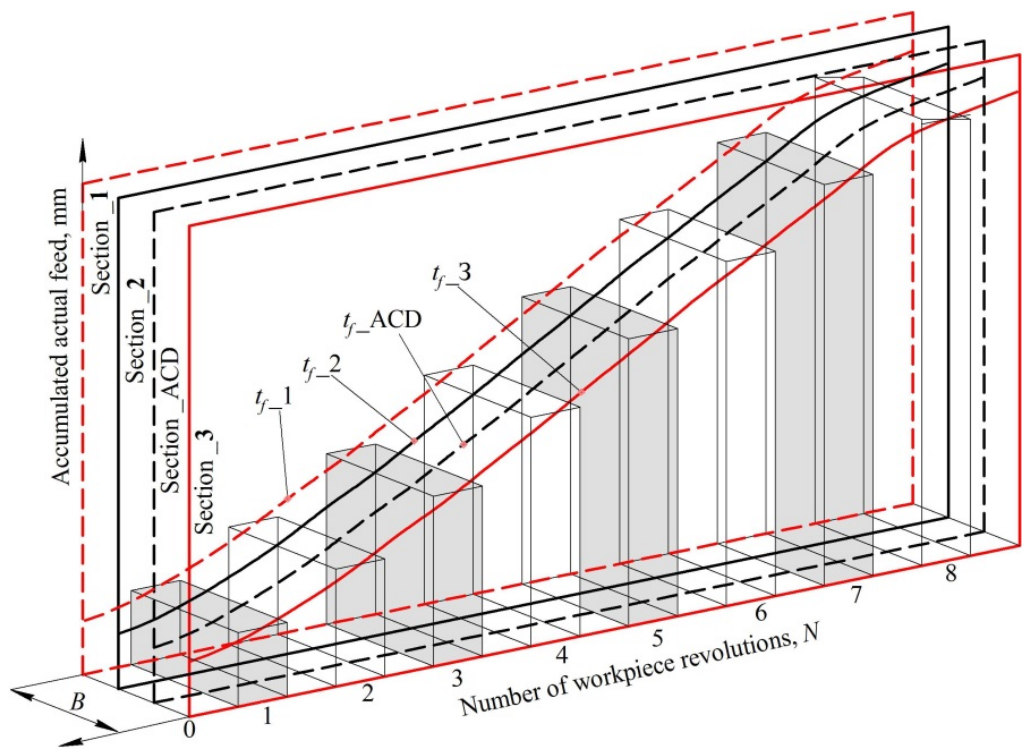

Fig. 4. Three-dimensional graph of the accumulated actual feed, constructed on each revolution of the workpiece in four shaft sections

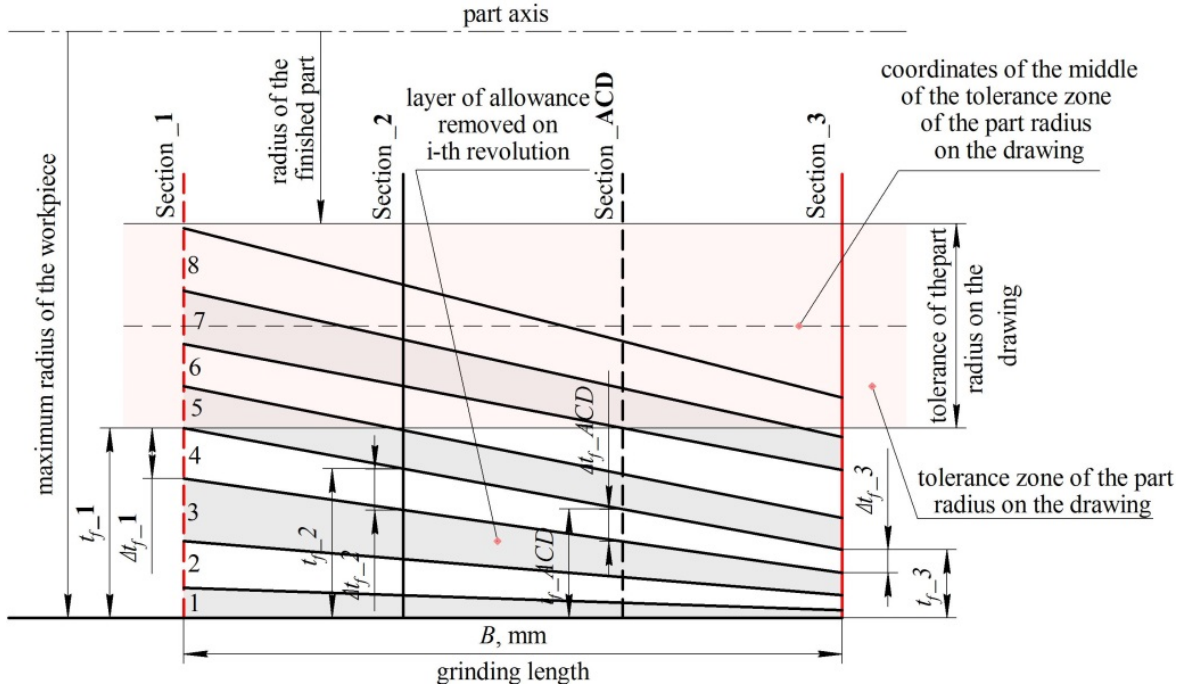

Fig. 5. Graphs of metal layers along the length of the treated surface B, constructed on each revolution of the workpiece in four shaft sections

\section{Conclusion}

1. Due to the absence in the modern CAD/CAM-systems the tool for designing optimal cycles of the cutting modes in real production conditions, the cutting modes for all types of the circular grinding operations with $\mathrm{CNC}$ are assigned manually by the technologist. At that the technologist relies only on personal experience or on the enterprise`s statistical data on cutting modes in similar operations. It should be noted separately that at the moment in the conditions of the automated production there are no tool for checking the developed 
grinding cycles for control programs to ensure the specified accuracy and other parameters of the treated surface' quality. Therefore at the moment predicting the processing accuracy in $\mathrm{CNC}$ circular grinding operations in which program feeds are changed in an automatic stepwise cycle is the difficult and unsolved scientific and technical task.

2. To solve the above problem, the DT of the circular plunge grinding operation with CNC is developed; this DT allows performing three-dimensional visualization of the forming process of the part treated surface by modeling the layer-by-layer metal removal along the entire length of the treated surface for different grinding conditions. The DT allows checking processing options of the part for different grinding conditions, correcting the cutting modes and improving the processing accuracy. As a result it becomes possible to use the developed DT as an applied tool for verification and preliminary checking of the designed cycle on providing the processing accuracy for the specified processing conditions by modeling the layer-by-layer allowance removal on each workpiece revolution considering the influence of variable factors. Introduction of the $\mathrm{CP}$ control on providing the processing accuracy reduces the reject and increases the operation' productivity, shortens the development and implementation of CP in production.

\section{References}

1. W.B Rowe Principles of Modern Grinding Technology (2009)

2. X.H. Le, H.K. Le, T.H. Tran, V.C. Nguyen, D.T. Do, H.P. Nguyen, A.T. Luu, P.V. Ngoc, J. LNNS. 104, 557 (2020)

3. J. Gu, H. Li, International Journal of Advanced Manufacturing Technology. 105, 7 (2019)

4. R. Gupta, K.S. Shishodia, G.S. Sekhon, J. Mater. Process. Technol. 112, 63 (2001)

5. S. Gao, C. Yang, J. Xu, Y. Fu, H. Su, W. Ding. International Journal of Advanced Manufacturing Technology. 92, 1105 (2017)

6. L. Tung, T. Hong, N. Cuong, N. Vu. Proceedings of the International Conference on Engineering Research and Applications. 104, 143 (2019)

7. D. Barrenetxea, J. Alvarez, J.I. Marquinez, I., Gallego, I.M., Perello, P. Krajnik International Journal of Machine Tools and Manufacture. 84, 17 (2014)

8. T.-H. Tran, A.-T. Luu, Q.-T. Nguyen, H.-K. Le, A.-T. Nguyen, T.-D. Hoang, X.-H. Le, T.-L. Banh, N.-P Vu. J. Lecture Notes in Networks and Systems. 9, 448 (2019)

9. V.N. Pi, L.X. Hung, L.A. Tung, B.T. Long. J. Mater. Sci. Eng. 6, 291 (2016)

10. L.X. Hung, V.N. Pi, L.A. Tung, H.X. Tu, G. Jun, B.T. Long. In: IOP Conference Series: Materials Science and Engineering. 417, 012 (2018)

11. S. Yudin, K. Smolyanoy, P. Pereverzev. IOP Conference Series Materials Science and Engineering. 0709, 033005 (2020)

12. P.P. Pereverzev, A.V. Popova, D.Yu. Pimenov J. Russian Engineering Research. 35, 215 (2015)

13. P.P. Pereverzev, A.V. Akintseva. J. Russian Engineering Research. 36, 1048 (2016)

14. A.V. Akintseva, A.V. Prokhorov, S.V. Omelchenko. IOP Conference Series Materials Science and Engineering. 709, 033003 (2020)

15. P.P. Pereverzev, A.V. Akintseva, M.K. Alsigar, D.V. Ardashev. Inter. Jour. of Mechanical Sciences. 139, 1 (2019) 